\title{
Design strategies to tune structural and mechanical properties of synthetic collagen hydrogels
}

Jinyuan Hu', Jie Wang', Xiaonan Zhu', Raymond S Tu', Vikas Nanda , Fei Xu'*

'Ministry of Education Key Laboratory of Industrial Biotechnology, School of Biotechnology, Jiangnan University, 214122 Wuxi, China

${ }^{2}$ Department of Chemical Engineering, The City College of City University of New York, 160 Convent Avenue, Steinman Hall T313, New York, New York 10031, United States

${ }^{3}$ Center for Advanced Biotechnology and Medicine and the Department of Biochemistry and Molecular Biology, Robert Wood Johnson Medical School, Rutgers, The State University of New Jersey, Piscataway, NJ 08854, United States

\section{Table of Contents}

Supplementary Methods: MALDI-TOF Mass Spectrometry.

Supplementary Tables and Figures

Table S1. The amino acid sequences of the designed collagen proteins

Figure S1. The standard curve of A275 and dry weight

Figure S2. The longitudinal and transverse cross sections schematic diagram and E3-VB-K3 hydrogel

Figure S3. Shearing force curves of the lyophilized gels of the designed proteins as labeled.

Figure S4. DLS of the particle size distribution and correlation coefficient for the designed collagen proteins

Figure S5. Storage and loss moduli G' and G', of the VB 


\section{Supplementary Methods}

MALDI-TOF Mass Spectrometry. UltrafleXtreme Matrix-Assisted Laser Desorption/ Ionization

Time of Flight (MALDI-TOF) was used to confirmed the designed collagen protein masses (Bruker, USA). The proteins were dissolved in deionized water and deposited on a $600 \mu \mathrm{m}$ AnchorChip target plate (Bruker), using $5 \mathrm{mg} / \mathrm{mL}$ 2,5-dihydroxyacetophenone, 25\% (v/v) ethanol, $1.5 \mathrm{mg} / \mathrm{mL}$ diammonium hydrogen citrate as matrix in linear mode, BSA was used for external calibration. VB m/z $=17399\left[(\mathrm{M}+\mathrm{H})^{1+}\right.$, calc. 17207], E3-VB-K3 m/z $=22698\left[(\mathrm{M}+\mathrm{H})^{1+}\right.$, calc. 22686]. E3-VBB-K3 m/z = 30313 [(M+H) ${ }^{1+}$, calc. 30319]. E3-VBBB-K3 m/z = 38970 [(M+H) ${ }^{1+}$, calc. 37952].

\section{Supplementary Tables and Figures}

Table S1. The amino acid sequences of the designed collagen proteins. The variable domain, V, is underlined, and the heterodimer-forming modules, E3 and K3, are highlighted in red.

\begin{tabular}{|c|c|}
\hline \multicolumn{2}{|c|}{ Amino acid Sequence } \\
\hline \multirow{3}{*}{$\stackrel{n}{>}$} & HHHHHHADEQEEKAKVRTELIQELAQGLGGIEKKNFPTLGDEDLDHTYMTKLLTYLQEREQA \\
\hline & ENSWRKRLLKGIQDHALDPGPRGEQGPQGLPGKDGEAGAQGPAGPMGPAGFPGERGEKGEPG \\
\hline & TQGAKGDRGETGPVGPRGERGEAGPAGKDGERGPVGPAG \\
\hline \multirow{4}{*}{ 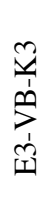 } & HHHHHHGEISALEKEISALEKEISALEKGGGGGGGADEQEEKAKVRTELIQELAQGLGGIEKKN \\
\hline & FPTLGDEDLDHTYMTKLLTYLQEREQAENSWRKRLLKGIQDHALDPGPRGEQGPQGLPGKDG \\
\hline & EAGAQGPAGPMGPAGFPGERGEKGEPGTQGAKGDRGETGPVGPRGERGEAGPAGKDGERGP \\
\hline & VGPAGGGGGGGGKISALKEKISALKEKISALKE \\
\hline \multirow{5}{*}{ 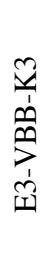 } & HHHHHHGEISALEKEISALEKEISALEKGGGGGGGADEQEEKAKVRTELIQELAQGLGGIEKK \\
\hline & NFPTLGDEDLDHTYMTKLLTYLQEREQAENSWRKRLLKGIQDHALDPGPRGEQGPQGLPGKD \\
\hline & GEAGAQGPAGPMGPAGFPGERGEKGEPGTQGAKGDRGETGPVGPRGERGEAGPAGKDGERG \\
\hline & PVGPAGPRGEQGPQGLPGKDGEAGAQGPAGPMGPAGFPGERGEKGEPGTQGAKGDRGETGP \\
\hline & VGPRGERGEAGPAGKDGERGPVGPAGGGGGGGGKISALKEKISALKEKISALKE \\
\hline
\end{tabular}



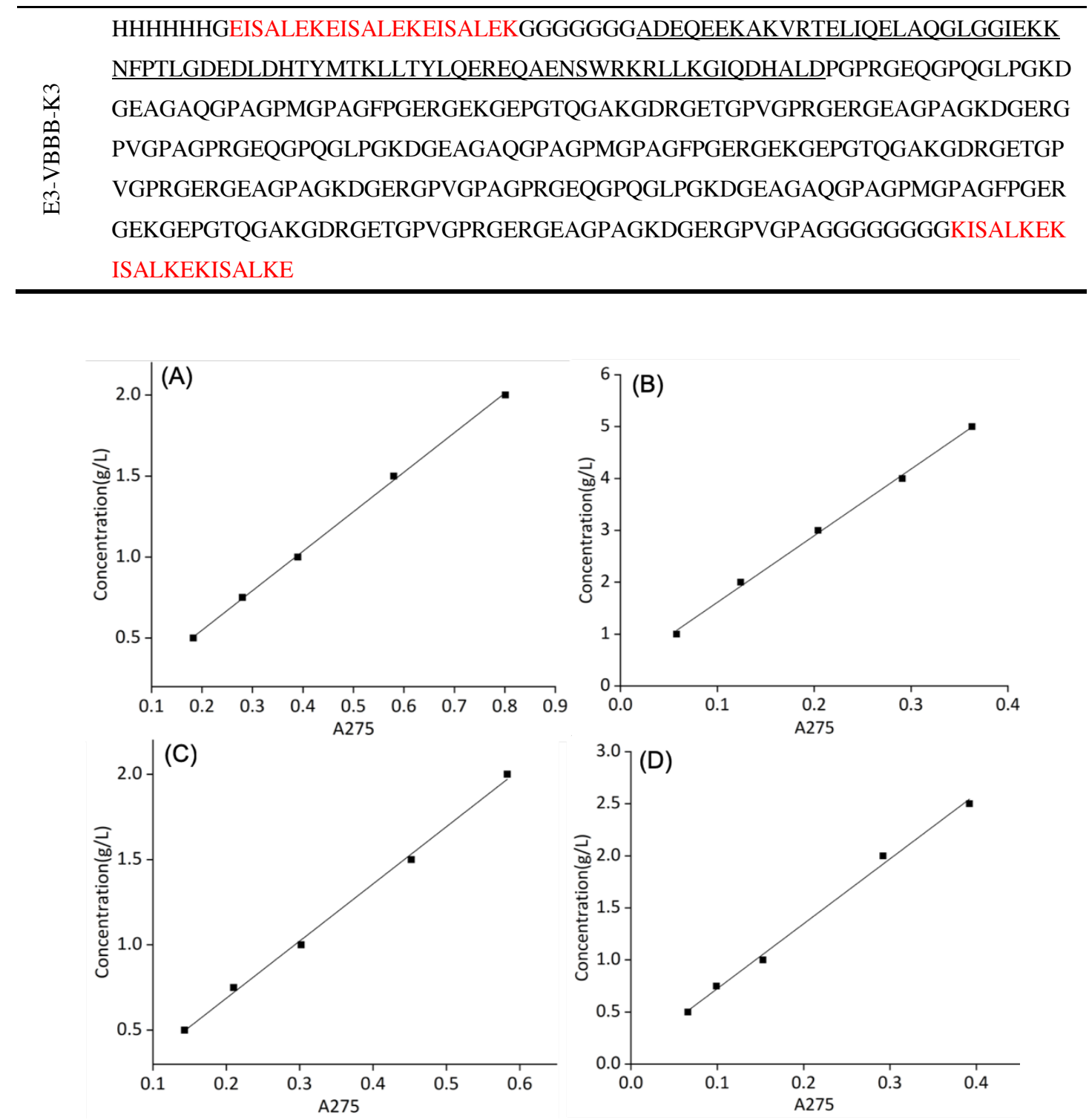

Figure S1. The standard curve of optical density at $275 \mathrm{~nm}$ (A275) and dry weight about the designed collagen proteins. (A) VB, (B) E3-VB-K3, (C) E3-VBB-K3, (D) E3-VBBB-K3.

(A)

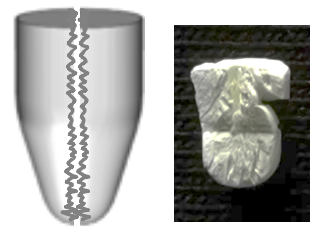

Longitudinal section
(B)

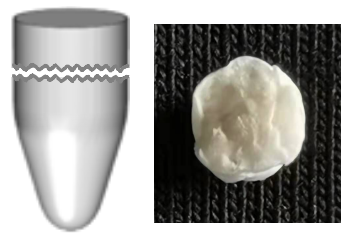

Cross section

Figure S2. The longitudinal (A) and transverse (B) cross sections schematic diagrams and images of a lyophilized E3-VB-K3 hydrogel. 


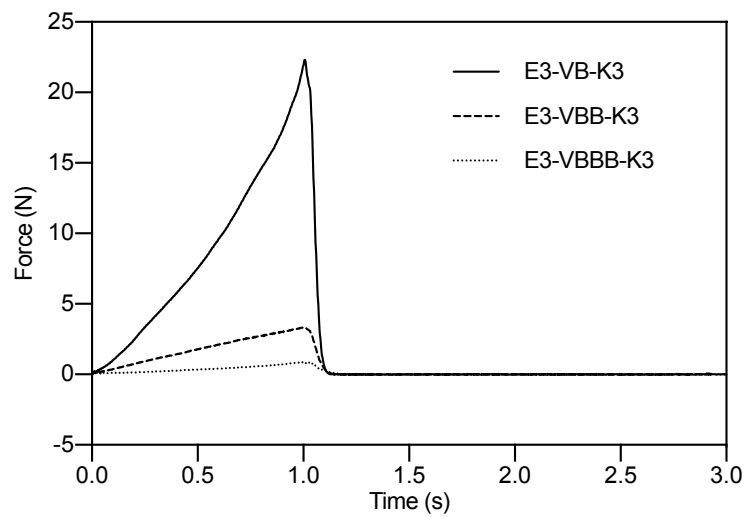

Figure S3. Shearing force curves of the lyophilized gels of the designed proteins as labeled. 


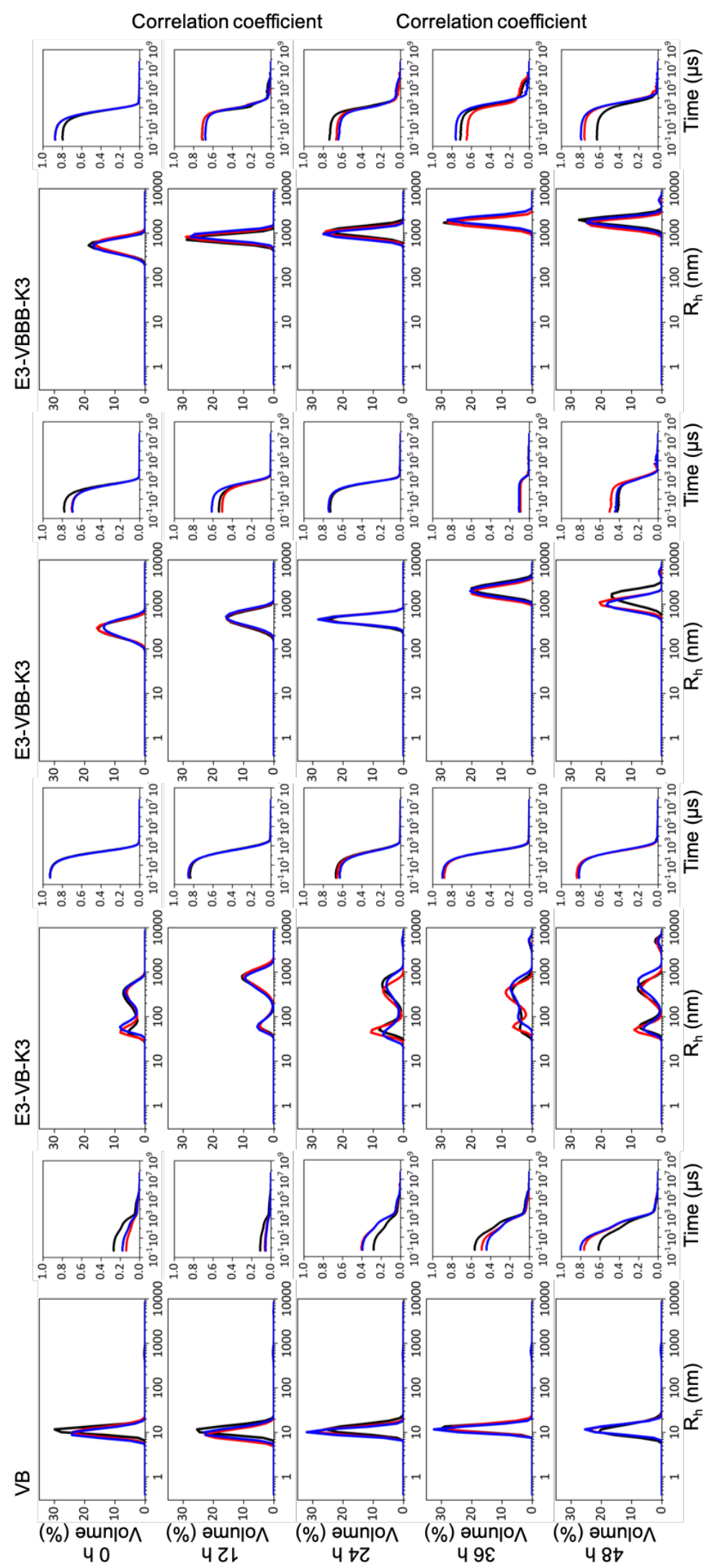

Figure S4. Dynamic Light Scattering (DLS) of the particle size distribution and correlation coefficient for the designed proteins detected with time at $4^{\circ} \mathrm{C}$. 

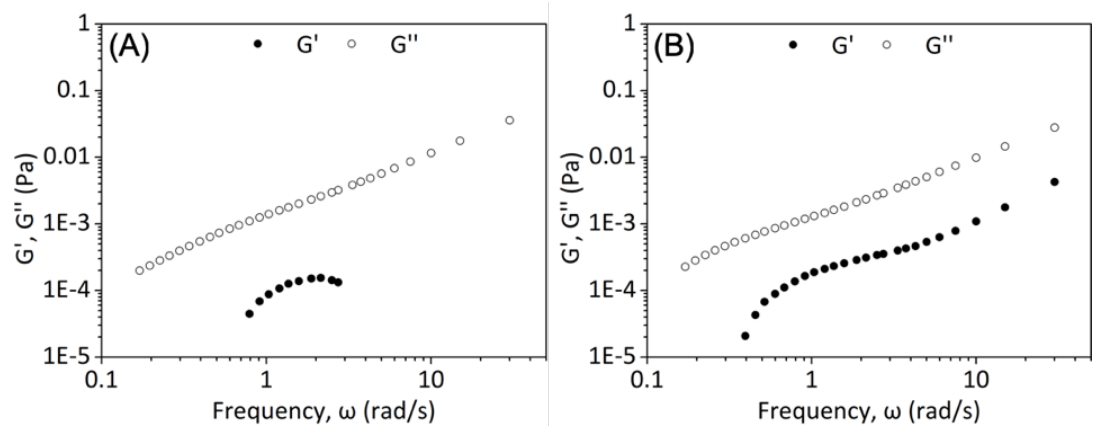

Figure S5. Storage and loss moduli G' and G' of the VB at (A) $0.50 \%$ and (B) $1.6 \%(\mathrm{w} / \mathrm{v})$. 